\title{
Effect of permissive hypercapnia on background cerebral electrical activity in premature babies
}

\author{
Suresh Victor ${ }^{1,2}$, Christopher M. McKeering ${ }^{3}$, Stephen A. Roberts ${ }^{4}$, Catherine Fullwood ${ }^{4,5}$ and Patrick A. Gaydecki ${ }^{3}$
}

\begin{abstract}
BACKGROUND: Permissive hypercapnia is routinely practiced in neonatal intensive care units. The effect of permissive hypercapnia on the preterm brain and brain electrical activity is unknown. In this study, we aimed to determine the effect of chronic changes in partial pressure of blood carbon dioxide $\left(\mathrm{PcO}_{2}\right)$ on brain electrical activity in preterm newborn babies born at or before 32 wk gestation.
\end{abstract}

METHODS: Eighty-three 1-h long digital electroencephalography (EEG) recordings were performed once a week for 4 wk on 25 babies with median gestational age of 29 wk (range: $23-32$ ) after $48 \mathrm{~h}$ of age. Capillary blood gas measurements were performed midway through EEG recordings.

RESULTS: There are associations between EEG parameters and blood $\mathrm{pH}, \mathrm{PcO}_{2}$, and blood glucose concentration. However, there are also strong and complex associations with gestational age and substantial individual patient effects that make it difficult to demonstrate predictive associations. $\mathrm{PcO}_{2}$ and bicarbonate are significantly correlated with relative power of $\theta E E G$ band and $\triangle E E G$ band respectively after adjustment for age and intrababy correlations, but after allowing for multiple testing these relationships are of borderline statistical significance.

CONCLUSION: Compensated respiratory acidosis may affect EEG by increased delta wave activity in preterm babies born before 32 wk gestation.

$\mathrm{H}$ ypocapnia before surfactant therapy in preterm infants has been associated with increased risk of bronchopulmonary dysplasia (1). As a result, permissive hypercapnia defined as partial pressure of blood carbon dioxide $\left(\mathrm{PcO}_{2}\right)$ of more than 7 $\mathrm{kPa}$ is routinely practiced in neonatal intensive care units (2).

The effect of hypercapnia on preterm brain has not been fully evaluated. A small randomized controlled trial showed that minimal ventilation with an aim to maintain $\mathrm{PcO}_{2}$ more than $7 \mathrm{kPa}$ showed an increased trend to adverse neurodevelopmental outcome (3). A review of two randomized controlled trials $(n=269)$ with no neurodevelopmental assessment did not demonstrate any significant overall benefit on mortality and pulmonary morbidity from permissive hypercapnia (4). An observational cohort study of 425 preterm infants which showed an increased risk of bronchopulmonary dysplasia with permissive hypercapnia did not evaluate the effect of hypercapnia on the preterm brain.

Electroencephalography (EEG) is the record of the brain electrical activity. Preterm EEG is discontinuous with bursts of high voltage slow wave electrical activity interspersed by periods of electrical quiescence called interburst interval (IBI) (5). Prolonged EEG IBIs of more than $30 \mathrm{~s}$ in preterm infants born at less than $28 \mathrm{wk}$ gestation and more than $25 \mathrm{~s}$ in preterm infants born at more $28 \mathrm{wk}$ gestation have been associated with increased incidence of death or developmental motor abnormalities $(6,7)$. EEG has been studied in relation to age, hypotension, hypo and hypercapnia, and cardiac output in preterm babies born before $30 \mathrm{wk}$ gestation during the first $72 \mathrm{~h}$ after birth (8-11). Lower levels of blood carbon dioxide have been associated with slowing of cerebral electrical activity and increased cerebral fractional oxygen extraction, while hypercapnia was associated with suppression of cerebral electrical activity during the first $48 \mathrm{~h}$ after birth (9). However, the incidence of hypercapnia, permissive or otherwise is more common (42.6\%) after $7 \mathrm{~d}$ of age than during the first $48 \mathrm{~h}$ after birth (2). No studies have been performed to evaluate the effect of hypercapnia on brain electrical activity in preterm infants born before $32 \mathrm{wk}$ gestational age after $72 \mathrm{~h}$ of age.

In this study, we aimed to determine the effect of chronic changes in $\mathrm{PcO}_{2}$ on brain electrical activity in preterm newborn babies born before $32 \mathrm{wk}$ gestation and more than $48 \mathrm{~h}$ of age along with a number of related parameters such as $\mathrm{pH}$ and bicarbonate.

\section{RESULTS}

Eighty-three EEG recordings were performed on 25 babies (11 males) with a median gestational age at birth of $29 \mathrm{wk}$ (range: 23-32) and median birth weight of $1,224 \mathrm{~g}$ (range: 635-1,695) at a single tertiary referral newborn intensive care unit. Their

\footnotetext{
'Institute of Human Development, University of Manchester, Manchester, UK; ${ }^{2}$ Central Manchester University Hospitals NHS Foundation Trust, The Manchester Academic Health Sciences Centre, Manchester, UK; ${ }^{3}$ School of Electrical and Electronic Engineering, University of Manchester, Manchester, UK; ${ }^{4}$ Centre for Biostatistics, Institute of Population Health, University of Manchester, The Manchester Academic Health Sciences Centre, Manchester, UK; ${ }^{5}$ Manchester Biomedical Research Centre, Central Manchester University Hospitals NHS Foundation Trust, The Manchester Academic Health Science Centre, Manchester, UK. Correspondence: Suresh Victor (Suresh.victor@manchester.ac.uk)
} 
median Apgar score at 5 min was 9 (range: 4-10). The condition of the babies at the time of EEG recording was as shown in Table 1. None of the babies were on inotropes, morphine, or antiepileptic medications at the time of the EEG recording. Only two babies had raised C-reactive protein at the time of recording. Only one baby had evidence of periventricular leukomalacia on follow-up cranial ultrasound scans.

There is a strong nonlinear trend in the EEG parameters with corrected gestational age (CGA) with a decrease in relative power of the $\alpha, \beta$, and $\theta$ EEG bands $\left(\mathrm{RP}_{\alpha}, \mathrm{RP}_{\beta}, \mathrm{RP}_{\theta}\right)$ to around $29 \mathrm{wk}$ and an increase thereafter (Figure 1). The relative power of the $\triangle$ EEG band $\left(\mathrm{RP}_{\Delta}\right)$ shows a similar effect but inverted. For all these parameters, the nonlinearity is highly significant $(P<0.001)$. In contrast, IBI shows a linear decrease with CGA. There are also lesser but clear differences between individual babies that are statistically significant (see below and Table 2)

$\mathrm{PcO}_{2}$ shows little change with gestational age, whereas $\mathrm{pH}$ and bicarbonate significantly increases with CGA and glucose level decreases (Figure 2).

$\mathrm{PcO}_{2}$ is significantly correlated with RP in all four frequency bands, but not with IBI (Figure 3; Table 2), and the association with $\mathrm{RP}_{\theta}$ remains significant $(P=0.013)$ after adjustment for age and intrababy correlations. However, this relationship is only of borderline significance after allowance is made for the fact that five frequency parameters have been assessed $(P=0.063)$. We observe that the relationship between $\mathrm{PcO}_{2}$ and $\mathrm{RP}_{\theta}$ remains highly significant after adjusting for CGA alone $(P=0.001)$ but is weakened after additionally allowing for individual patient differences. Glucose and $\mathrm{pH}$ have significant correlations with EEG parameters, but these are lost on adjustment for CGA. In contrast, bicarbonate levels show significant associations with EEG parameters only after adjusting for CGA, but the relationship between $\mathrm{RP}_{\Delta}$ and bicarbonate would be considered as significant even after adjustment for the multiple testing $(P=0.039)$. As shown in Table 2 , the CGA effect remains highly significant for all EEG components after adjustment for each of the clinical variables, and (with one exception) the interpatient variability likewise remains significant.

Two babies died before discharge. Of the 23 survivors, 9 (39\%) babies had chronic lung disease defined as oxygen requirement at 36 wk CGA. Nine babies did not complete neurodevelopment follow-up. Of the remaining 14 babies, 12 infants were noted to have neurodevelopment within normal limits at $2 \mathrm{y}$

Table 1. Median (range) of corrected gestational age, age, $\mathrm{pH}, \mathrm{PcO}_{2}$, blood glucose concentration, and bicarbonate at the time of EEG recordings

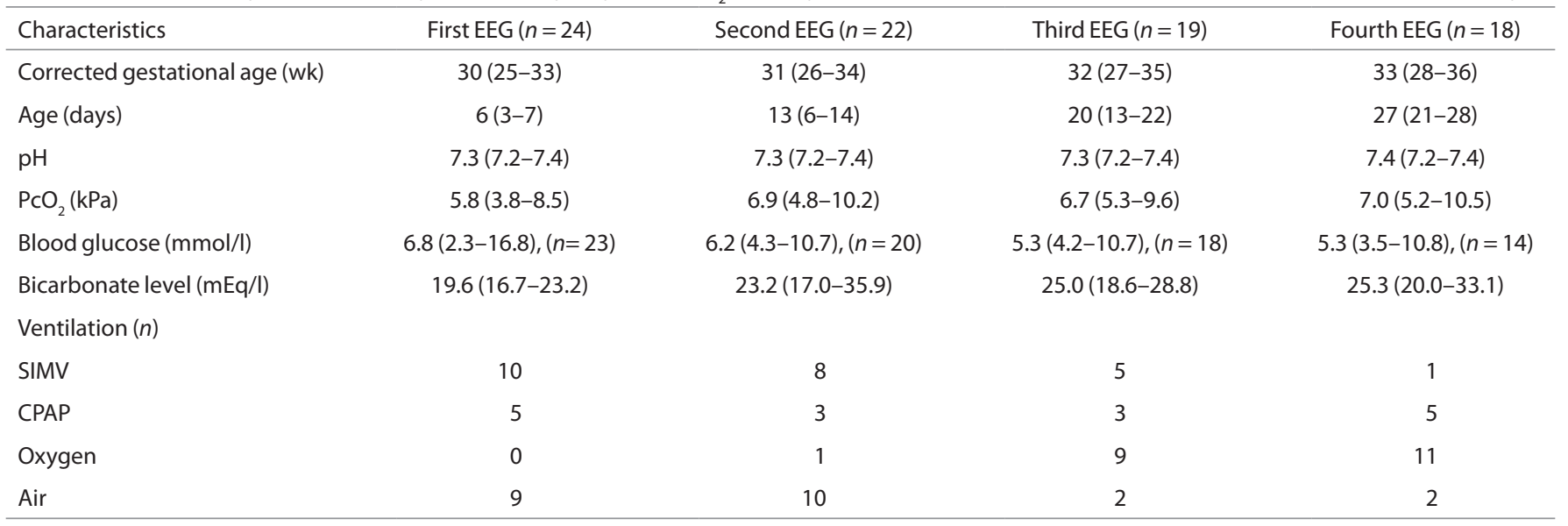

CPAP, continuous positive airway pressure; EEG, electroencephalography; SIMV, synchronized intermittent mandatory ventilation.

a

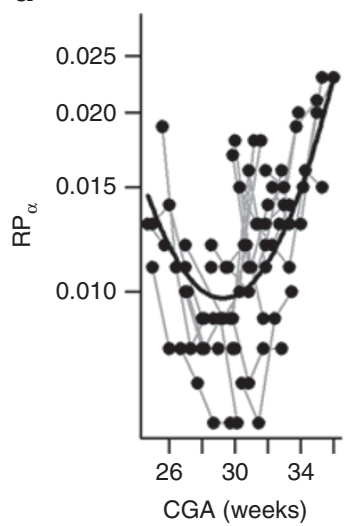

b

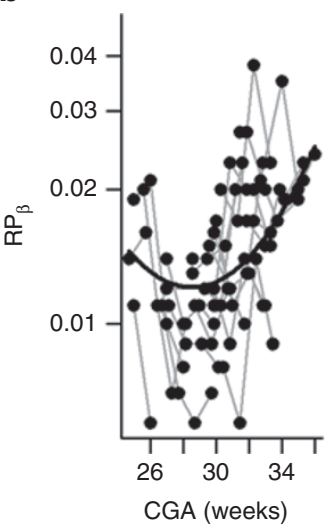

c

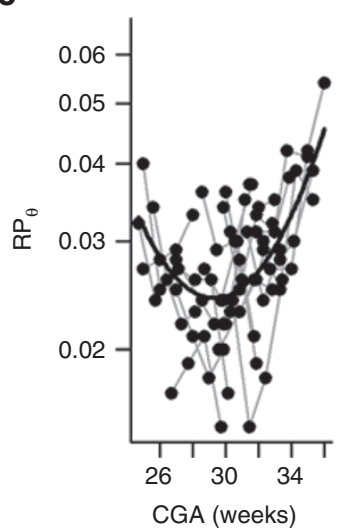

d

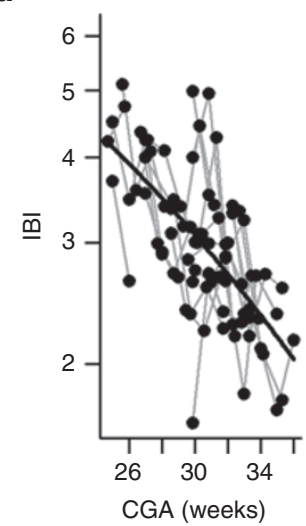

e

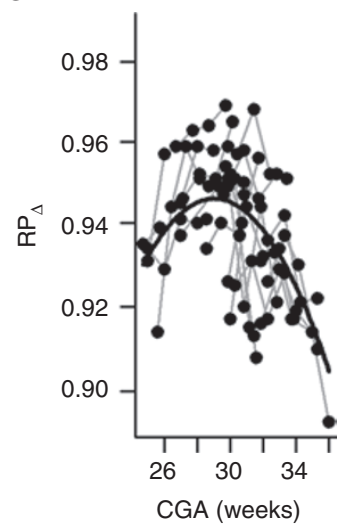

Figure 1. Relationship between electroencephalography parameters and corrected gestational age (CGA). (a) $\mathrm{RP}_{\alpha^{\prime}}(\mathbf{b}) \mathrm{RP}_{\beta^{\prime}}(\mathbf{c}) \mathrm{RP}_{\theta^{\prime}}(\mathbf{d})$ interburst interval, and (e) $\mathrm{RP}_{\Delta}$. Grey lines join repeat measurements from the same babies. Solid line is a regression fit using a quadratic function. 
Table 2. Associations between clinical and EEG variables

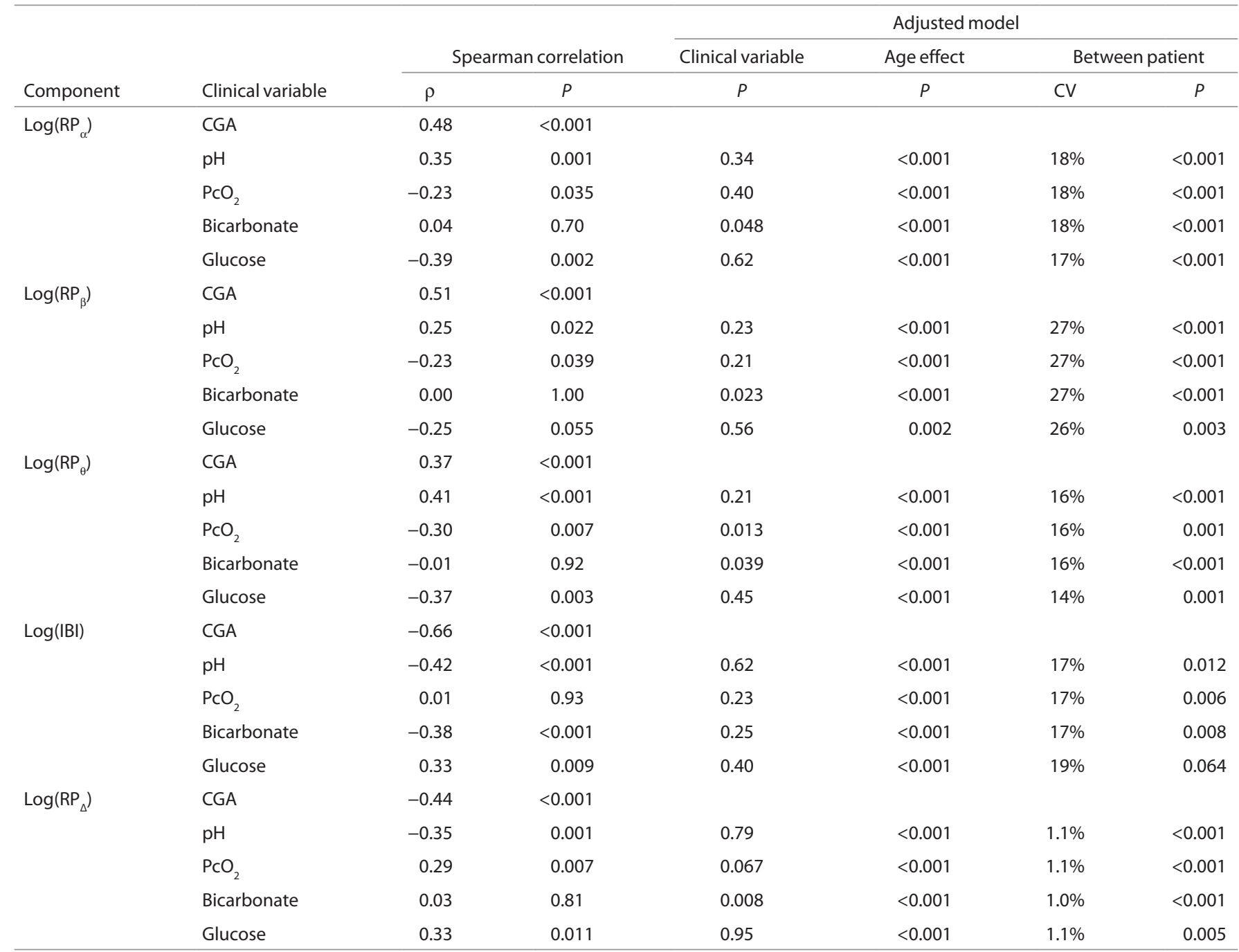

Uncorrected Spearman correlations and an adjusted model allowing for a quadratic age effect and intrapatient correlations. The adjusted models for each EEG parameter included effects of the clinical variable, an age effect and a patient effect, and $P$ values are given for each effect along with the between-patient CV. $P$ values in the table are not adjusted for multiple testing (but see text).

CGA, corrected gestational age; CV, coefficient of variation; EEG, electroencephalography; IBI, interburst interval.

of age, $1 \mathrm{infant}$ had mild neurodevelopment delay (gestation at birth: $31 \mathrm{wk}$; twin pregnancy; normal cranial ultrasound scan; no chronic lung disease; $\mathrm{PcO}_{2}$ range: $5.4-8.1 \mathrm{kPa}$; IBI range: 2.6-5.3 s), and 1 infant had moderate neurodevelopment delay (gestation at birth: $29 \mathrm{wk}$; singleton pregnancy; parieto-occipital periventricular leukomalacia on cranial ultrasound scan; no chronic lung disease; $\mathrm{PcO}_{2}$ range: $5.3-6.2 \mathrm{kPa}$; IBI range: $1.6-3.3 \mathrm{~s})$.

\section{DISCUSSION}

Our results show that there are associations between EEG and $\mathrm{pH}, \mathrm{PcO}_{2}$, bicarbonate, and glucose. However, there are also strong and quite complex associations with gestational age and substantial individual patient effects that make it difficult to demonstrate predictive associations within individual patients. Nevertheless, $\mathrm{PcO}_{2}$ is correlated with $\mathrm{RP}_{\theta}$ after adjustment for age and intrababy correlations although this effect is of borderline significance after allowing for multiple testing. There is also an association between blood bicarbonate concentrations and $\mathrm{RP}_{\Delta}$, which would be considered significant after all adjustments. In carefully adjusting for CGA and for interpatient variability, the analyses here are conservative and real effects of $\mathrm{PcO}_{2}$ and bicarbonate will have been absorbed into the age and interpatient effects. Taken as a whole, these data suggest that there may be associations between EEG parameters and compensated respiratory acidosis in babies more than $48 \mathrm{~h}$ of age, which warrant further study, but the data here do not allow us to draw any firm conclusions about specific EEG and clinical parameters.

Changes in $\mathrm{RP}_{\theta}$ in relation to $\mathrm{PcO}_{2}$ are likely to be due to the inverse effect of the changes in $\mathrm{RP}_{\Delta} \cdot \mathrm{RP}_{\Delta}$ increases with increase in $\mathrm{PcO}_{2}$ (not statistically significant) and increase in blood bicarbonate concentrations (statistically significant). As $39 \%$ of our cohort had chronic lung disease, the changes in $\mathrm{PcO}_{2}$ and bicarbonate are likely to be chronic. Together, this indicates an increased level of EEG $\Delta$ wave activity with 

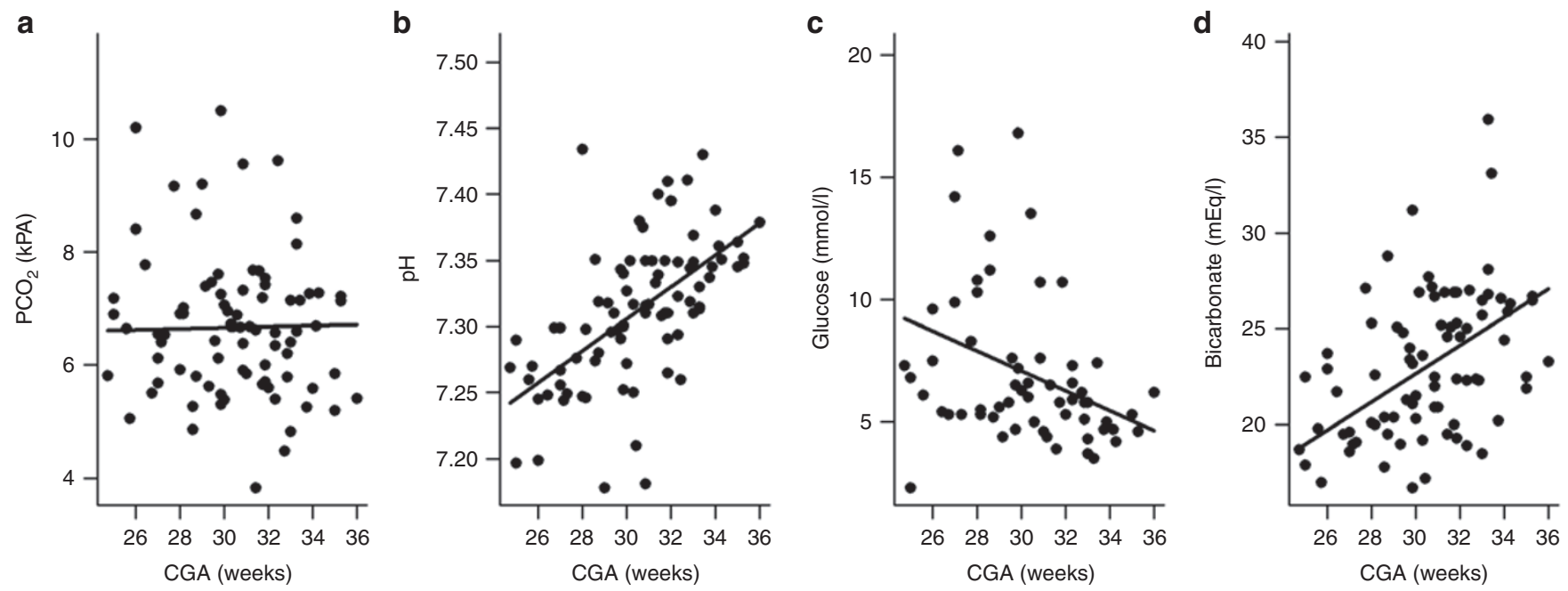

Figure 2. Relationship between clinical parameters and corrected gestational age (CGA). Solid lines are a linear regression fit. (a) $\mathrm{PcO},(P=0.76),(\mathbf{b}) \mathrm{pH}$ $(P<0.001)$, (c) glucose $(P=0.014)$, and $(\mathbf{d})$ bicarbonate $(P<0.001)$.

a

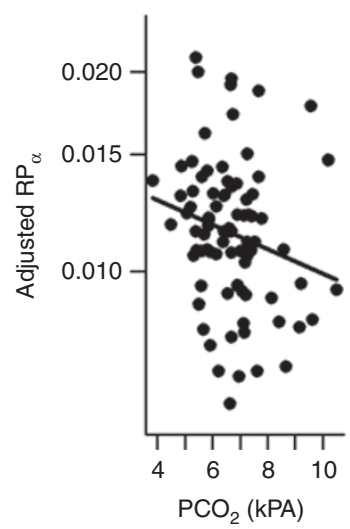

b

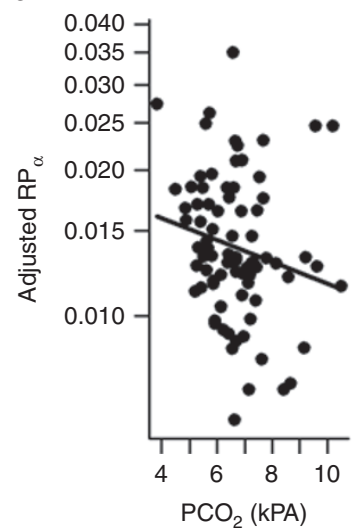

C

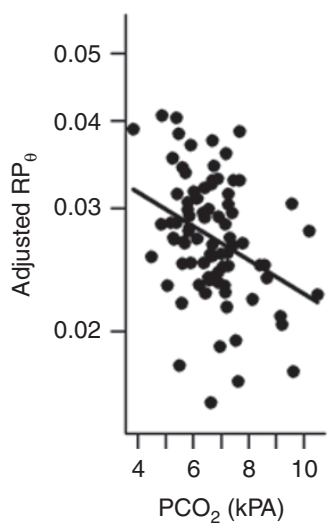

d

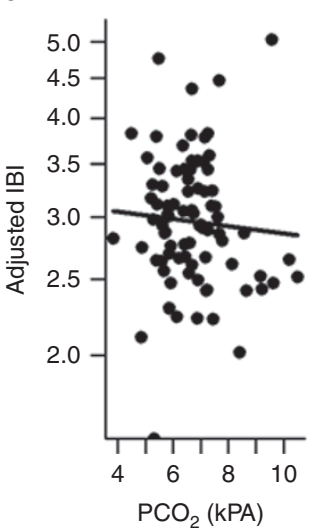

e

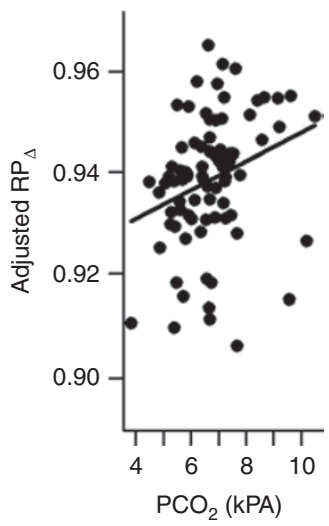

Figure 3. Relationship between electroencephalography parameters and $\mathrm{PcO}_{2}$ levels after adjustment for corrected gestational age (quadratic fit) and interbaby effects. Solid lines are a linear regression fit. (a) $\mathrm{RP}_{\alpha}(P=0.40),(\mathbf{b}) \mathrm{RP}_{\beta}(P=0.21),(\mathbf{c}) \mathrm{RP}_{\theta}(P=0.013),(\mathbf{d})$ interburst interval (IBI) $(P=0.23)$, and $(\mathbf{e}) \operatorname{RP}_{\Delta}(P=0.07)$.

compensated respiratory acidosis. Indeed, Scher et al. (12) showed that babies with chronic lung disease had a significant reduction in the percentage of active sleep. In addition, they noted significant differences in relation to the number $(0.29 /$ min vs. $0.13 / \mathrm{min}$ ) and duration (4.8 vs. $2.94 \mathrm{~s}$ ) of arousals as well as the total number of body movements $(1.57 / \mathrm{min}$ vs. $0.74 / \mathrm{min}$ ) (12). These data suggest that chronic changes in blood gases in babies more than $48 \mathrm{~h}$ old may be associated with unfavorable EEG background warranting further study on the effects of permissive hypercapnia on the preterm brain.

The effects of $\mathrm{pH}, \mathrm{PcO}_{2}$, and blood glucose concentration on EEG when babies are more than $48 \mathrm{~h}$ old differ from those noted in previous studies conducted during the first $48 \mathrm{~h}$ after birth. Victor et al. (9) showed that lower levels of $\mathrm{PcO}_{2}$ were associated with increased cerebral fractional oxygen extraction and slowing of EEG, while higher levels of $\mathrm{PcO}_{2}$ were associated with suppression of EEG during the first $48 \mathrm{~h}$ of age. Granot et al. (13) using amplitude integrated EEG on 65 preterm babies born before $28 \mathrm{wk}$ gestational age showed that respiratory acidosis and hyperglycemia during the first $72 \mathrm{~h}$ after birth was associated with suppressed electrical activity. Wikström et al. (14) using one-channel EEG reported that in a cohort of 32 babies born before $28 \mathrm{wk}$ gestation higher levels of $\mathrm{PcO}_{2}$ were associated with suppressed cerebral electrical activity. Wikström et al. (14) showed that high plasma glucose and low plasma glucose were both associated with suppressed electrical activity during the first $72 \mathrm{~h}$ after birth. Eaton et al. (15) using continuous EEG monitoring conducted from 3.5 to $165 \mathrm{~h}$ of age showed that metabolic and respiratory acidocis were associated with prolonged IBIs. It appears that the preterm brain responds differently to acute and chronic changes in $\mathrm{PcO}_{2}$ with suppression of EEG activity with acute hypercapnia and increased EEG activity with chronic hypercapnia.

The strong trends in EEG parameters in relation to CGA noted in our cohort are consistent with the results of other studies $(16,17)$. Indeed, in our study, IBIs decrease with increased CGA (17). In our study, $\mathrm{RP}_{\Delta}$ shows slight increase until $30 \mathrm{wk}$ gestational age possibly due to the decrease in IBI 
and thereafter decreases sharply possibly due to an overall increase in frequency and decrease in amplitude of the EEG waves. Much of the changes in the relative power of other EEG bands $(\theta, \alpha$, and $\beta)$ are more likely to be due to the inverse of the effects of $\mathrm{RP}_{\Delta}$ than due to absolute changes in the EEG bands themselves.

The nonlinear energy operator method was used to detect bursts as has been shown in previous publications (18). However, we performed the EEG burst detection optimization using our own IBI markings. We found that by performing our own independent markings along with burst detection and artifact removal, we were able to achieve an automated burst detector that ascertained interburst values. When comparing the averaged values of the IBIs against the gestational ages of the babies, a strong and significant regression was confirmed.

The artifact detection consisted of a method similar to that which detected bursts. The method used a threshold that was based on signal epochs that were agreed to be artifact between two clinical neurophysiologists. Therefore, EEG signals that were both large in amplitude and high in frequency could be detected automatically using this method. The relative powers were detected using a window length of $2 \mathrm{~s}$ with a $1 \mathrm{~s}$ overlap.

The strong relationships with gestational age and substantial individual patient variability in EEG measurements in this study, combined with the large interval between measurements make it difficult to robustly demonstrate effects of $\mathrm{PcO}_{2}$ and related variables on the EEG parameters. Here, in the analysis, we have been conservative in only claiming effects which led to significant associations after adjustment for CGA and patient. Since the clinical parameters themselves are confounded with CGA, this adjustment is likely to be overconservative. The results here suggest that further work is required with shorter time periods between measurements and with more measurements per baby, which will allow comparisons within the same babies at essentially the same gestational age.

In conclusion, this study suggests that compensated respiratory acidosis affect EEG in preterm babies born before $32 \mathrm{wk}$ gestation and more than $48 \mathrm{~h}$ old. The suppression of EEG noted in acute respiratory acidosis is not seen in chronic compensated respiratory acidosis. Further research is required in assessing the effects of permissive hypercapnia on preterm brain injury.

\section{METHODS}

A prospective observational study was performed at the Newborn Intensive Care unit, St Mary's Hospital for Women and Children, Manchester, UK. The study was approved by the Oldham Local Research Ethics Committee. Infants born at or before $32 \mathrm{wk}$ gestation were recruited to the study following informed parental consent. Infants with significant intraventricular hemorrhages (defined as hemorrhages extending beyond the germinal matrix) have abnormally prolonged periods of EEG discontinuity and were therefore excluded (19).

Digital EEG recordings were performed for $60 \mathrm{~min}$ once a week for up to 4 wk with the first EEG recording being at least $48 \mathrm{~h}$ postbirth. The electrodes were placed at $\mathrm{Fp} 1, \mathrm{C} 3, \mathrm{~T} 3, \mathrm{O} 1, \mathrm{Fp} 2, \mathrm{C} 4, \mathrm{~T} 4, \mathrm{O} 2$, and $\mathrm{Cz}$ by trained clinical neurophysiologists. Disposable cup-shaped silver-silver chloride electrodes were placed on the scalp and secured using EEG paste.
The nine channels were arranged into a standard 12 signal observation pattern. These signals included the following channel differences: Fp2-T4, T4-O2, Fp2-C4, C4-O2, Fp1-T3, T3-O1, Fp1-C3, C3-O1, $\mathrm{T} 4-\mathrm{C} 4, \mathrm{C} 4-\mathrm{Cz}, \mathrm{Cz}-\mathrm{C} 3$, and $\mathrm{C} 3-\mathrm{T} 3$.

These 12 signals were used for all channel EEG analysis. The sampling frequency of the signals, across all channels, was $200 \mathrm{~Hz}$. EEG was automatically analyzed to determine the relative powers of EEG frequency bands, EEG IBI, and the presence of artifact.

\section{Relative Powers of EEG Frequency Bands}

The relative powers of selected EEG frequency bands were calculated with an initial fourth order high pass Butterworth IIR filter with a cut off of $0.3 \mathrm{~Hz}$ in place. The length of the reduced relative power window was 400 samples with a window overlap of 200 samples. A Hanning window was convolved with each signal epoch to reduce spectral leakage between frequency bins. The relative powers were calculated by dividing the value of their associated frequency range by the total of the frequency bins up to and including $30 \mathrm{~Hz}$. The spectrum was divided as follows: $\Delta$ band $(0.5-3.5 \mathrm{~Hz}), \theta$ band $(4-7.5 \mathrm{~Hz}), \alpha$ band $(8-12.5$ $\mathrm{Hz})$, and $\beta$ band $(13-30 \mathrm{~Hz})$. The relative power values were calculated for each individual channel, and the mean of the values was found by averaging over all 12 channels. The median relative power values over the entire $1 \mathrm{~h}$ EEG recording were used for statistical analysis to account for sleep-wake cycling and minimize the effects of handling for blood sampling on EEG. Relative power values that contained signal artifact (as defined below) were not included in the calculation of the median.

\section{IBIs}

A fourth order high pass Butterworth IIR filter with a cut off of 0.4 $\mathrm{Hz}$ and a sixth order low pass Butterworth IIR filter with a $30 \mathrm{~Hz}$ cutoff were initially applied to the EEG channels. The EEG IBIs were calculated by using the nonlinear energy operator method and the equation $E(n)=x(n) x(n-3)-x(n-1) x(n-2)$, where $E(n)$ is the operator and $x(n)$ is the input signal (20). A smoothing window of 203 samples was applied to the nonlinear energy operator values to obtain an overall value of $E(k)$. An $E(k)$ value greater than 5,932 was used to determine whether a burst was occurring. A minimum criterion of $1 \mathrm{~s}$ in length was defined for burst and IBIs. The median IBI over the entire $1 \mathrm{~h}$ EEG recording was used for statistical analysis to account for sleep-wake cycling. IBI values that contained signal artifact were not included in the calculation of the median.

\section{Artifact Detection}

The artifact detection was performed using a method similar to the burst detection. However, a fourth order Butterworth IIR band stop filter was used with cutoff frequencies of 49 and $51 \mathrm{~Hz}$ to remove noise. Additionally, a different equation was used for artifact detection as compared to the burst detection nonlinear energy operator method, $E(n)=x(n)^{2}-x(n+1) x(n-1)$, where $E(n)$ is the operator and $x(n)$ is the input signal (21). A smoothing window of 203 samples with an $E(k)$ threshold of $2.88949 \times 10^{6}$ was used. An artifact detection would cause the burst detection process to be paused while an artifact was occurring. Burst detection and interburst length counting continued once the artifact was no longer present.

\section{Clinical Measurements}

The primary measure, $\mathrm{PcO}_{2}$, along with secondary measures $\mathrm{pH}$, bicarbonate, and blood glucose concentration using capillary blood were measured midway through the EEG recording using ABL 835 Flex (Radiometer, Copenhagen, Denmark). Cranial ultrasound scans were performed as deemed clinically necessary until discharge from the neonatal unit. The images from these scans were examined for periventricular leukomalacia.

\section{Clinical Management}

The clinical management of the babies in this study was according to local clinical guidelines. In babies more than $48 \mathrm{~h}$ old, $\mathrm{PcO}_{2}$ was allowed to increase, provided the $\mathrm{pH}$ was maintained above 7.2. If two consecutive blood glucose measurements more than $2 \mathrm{~h}$ apart were more than $12 \mathrm{mmol} / \mathrm{l}$, insulin infusion was commenced to maintain blood glucose concentrations below $12 \mathrm{mmol} / \mathrm{l}$. 
A formal neurodevelopmental assessment was not performed. However, follow-up was offered by the clinical team until $2 \mathrm{y}$ of age. The outcome of this follow-up was recorded.

\section{Statistical Methods}

Correlations between clinical and EEG variables were assessed using Spearman correlations. Mixed linear models were used to assess the associations between EEG and clinical variables, with the EEG variables being log-transformed. CGA in weeks was included as a quadratic function, and the significance of the nonlinearity tested by comparison with a linear representation using likelihood ratio tests. Patient identifiers were added as random effects to allow for correlations within babies, and the magnitude of the interbaby differences expressed as coefficient of variation (CV). Significance of these effects was assessed using a restricted likelihood ratio test based on simulated values from the finite posterior distribution (22). As five EEG parameters were tested, the Holm test was used to generate multiplicity-adjusted $P$ values. Models were fitted using R (23) and the lme4 and RLRsim (22) packages.

\section{ACKNOWLEDGMENTS}

The authors acknowledge the technical assistance provided by Neurophysiology Department, Central Manchester University Hospitals NHS Foundation Trust. Support in recruitment and data collection was provided by Anna Hendrickson (research nurse), Akash Raina (consultant neonatologist) and Mohammed Sarwar (consultant neonatologist).

\section{STATEMENT OF FINANCIAL SUPPORT}

This work was funded by University of Manchester Intellectual Property and Manchester Integrating Medicine and Innovative Technology and facilitated by the Manchester Biomedical Research Centre.

Disclosure: None of the authors have any conflict of interest in regard to this article.

\section{REFERENCES}

1. Garland JS, Buck RK, Allred EN, Leviton A. Hypocarbia before surfactant therapy appears to increase bronchopulmonary dysplasia risk in infants with respiratory distress syndrome. Arch Pediatr Adolesc Med 1995;149:617-22.

2. van Kaam AH, De Jaegere AP, Rimensberger PC; Neovent Study Group. Incidence of hypo- and hyper-capnia in a cross-sectional European cohort of ventilated newborn infants. Arch Dis Child Fetal Neonatal Ed 2013;98:F323-6.

3. Thome UH, Ambalavanan N. Permissive hypercapnia to decrease lung injury in ventilated preterm neonates. Semin Fetal Neonatal Med 2009;14:21-7.

4. Woodgate PG, Davies MW. Permissive hypercapnia for the prevention of morbidity and mortality in mechanically ventilated newborn infants. Cochrane Database of Systematic Reviews 2001;CD002061.

5. Scher MS. Electroencephalography of the newborn: normal and abnormal features. In: Niedermeyer E, Da Silva FL, eds. Electroencephalography: Basic Principles, Clinical Applications, and Related Fields. 4th edition. Baltimore: Williams and Wilkins, 1999: 896-946.

6. Hayakawa F, Okumura A, Kato T, Kuno K, Watanabe K. Dysmature EEG pattern in EEGs of preterm infants with cognitive impairment: maturation arrest caused by prolonged mild CNS depression. Brain Dev 1997;19: $122-5$.
7. Marret S, Parain D, Jeannot E, Eurin D, Fessard C. Positive rolandic sharp waves in the EEG of the premature newborn: a five year prospective study. Arch Dis Child 1992;67:948-51.

8. Victor S, Appleton RE, Beirne M, Marson AG, Weindling AM. Spectral analysis of electroencephalography in premature newborn infants: normal ranges. Pediatr Res 2005;57:336-41.

9. Victor S, Appleton RE, Beirne M, Marson AG, Weindling AM. Effect of carbon dioxide on background cerebral electrical activity and fractional oxygen extraction in very low birth weight infants just after birth. Pediatr Res 2005;58:579-85.

10. Victor S, Marson AG, Appleton RE, Beirne M, Weindling AM. Relationship between blood pressure, cerebral electrical activity, cerebral fractional oxygen extraction, and peripheral blood flow in very low birth weight newborn infants. Pediatr Res 2006;59:314-9.

11. Victor S, Appleton RE, Beirne M, Marson AG, Weindling AM. The relationship between cardiac output, cerebral electrical activity, cerebral fractional oxygen extraction and peripheral blood flow in premature newborn infants. Pediatr Res 2006;60:456-60.

12. Scher MS, Richardson GA, Salerno DG, Day NL, Guthrie RD. Sleep architecture and continuity measures of neonates with chronic lung disease. Sleep 1992;15:195-201.

13. Granot S, Meledin I, Richardson J, Friger M, Shany E. Influence of respiratory acidosis and blood glucose on cerebral activity of premature infants. Pediatr Neurol 2012;47:19-24.

14. Wikström S, Lundin F, Ley D, et al. Carbon dioxide and glucose affect electrocortical background in extremely preterm infants. Pediatrics 2011;127:e1028-34.

15. Eaton DG, Wertheim D, Oozeer R, Dubowitz LM, Dubowitz V. Reversible changes in cerebral activity associated with acidosis in preterm neonates. Acta Paediatr 1994;83:486-92.

16. Bell AH, McClure BG, McCullagh PJ, McClelland RJ. Variation in power spectral analysis of the EEG with gestational age. J Clin Neurophysiol 1991;8:312-9.

17. Hayakawa M, Okumura A, Hayakawa F, et al. Background electroencephalographic (EEG) activities of very preterm infants born at less than 27 weeks gestation: a study on the degree of continuity. Arch Dis Child Fetal Neonatal Ed 2001;84:F163-7.

18. Palmu K, Wikström S, Hippeläinen E, Boylan G, Hellström-Westas L, Vanhatalo S. Detection of 'EEG bursts' in the early preterm EEG: visual vs. automated detection. Clin Neurophysiol 2010;121:1015-22.

19. Benda GI, Engel RC, Zhang YP. Prolonged inactive phases during the discontinuous pattern of prematurity in the electroencephalogram of very-low-birthweight infants. Electroencephalogr Clin Neurophysiol 1989;72:189-97.

20. Plotkin EI, Swamy MNS. Nonlinear Signal Processing Based on Parameter Invariant Moving Average Modeling. In Proceedings CCECE’21, Toronto, Canada, September 1992: TM3.11.1-TM3.11.4.

21. Kaiser JF. On a Simple Algorithm to Calculate the Energy of a Signal. In Proceeding of: Acoustics, Speech, and Signal Processing, 90, Vol 1 1990;381-4.

22. Scheipl F, Greven S, Kuechenhoff H. Size and power of tests for a zero random effect variance or polynomial regression in additive and linear mixed models. Comput Stat Data An 2008;52:3283-99.

23. R Core Team. R: a language and environment for statistical computing. $\mathrm{R}$ Foundation for Statistical Computing, 2013. http://www.r-project.org. 